\title{
An Analysis of the Folded Waveguide: A Compact Waveguide Launcher For ICRF Heating
}

\author{
B. M. JOST AND J. E. SCHARER
}

\begin{abstract}
Both theoretical and experimental investigations of the folded waveguide launcher as proposed for use in the ion-cyclotron range of frequencies (ICRF) heating of tokamak plasmas are presented. Theoretical results include an analysis of the folded waveguide using Ritz's method to obtain various field quantities. The cutoff wavenumbers from this analysis are within $10 \%$ of those calculated using an unfolded equivalent rectangular waveguide model. Measurements of cavity resonances for a scale model ICRF-folded waveguide with a cutoff frequency of $2.4 \mathrm{GHz}$ also agree closely with those calculated using an unfolded equivalent model. An unfolded equivalent waveguide model is used to evaluate properties of an inductive strip placed in the folded waveguide. Applying variational analyses provides upper and lower bounds to the reflection coefficient magnitude $\left|S_{11}\right|$ that are in good agreement with measured values. Next, polarizing plates (which have been proposed to increase the on-axis directivity of the radiated power) are modeled using an unfolded equivalent waveguide. A variational analysis is used to determine an upper-bound value of $\left|S_{11}\right|$ in air due to a polarizing plate inside a matched folded waveguide. Measured values are found to be within $3 \%$ of the calculated values, with $\left|S_{11}\right|>0.95$ for frequencies between 2.5 and $4.5 \mathrm{GHz}$. Finally, farfield radiation patterns from the folded waveguide are obtained at $\mathbf{3 . 0}$ $\mathrm{GHz}$ in air for both an open-ended folded waveguide and a folded waveguide with a polarizing plate attached; these measurements are compared with theoretical analyses and are found to be in good agreement. It is found that the free-space far fields of the open-ended folded waveguide are dominated by those originating at the fin ends, leading to radiation patterns which can be modeled using the equivalent unfolded rectangular waveguide far fields.
\end{abstract}

\section{INTRODUCTION}

$\mathrm{T}$ HE USE of folded waveguides for the propagation of electromagnetic energy dates back to the early 1940 's, when the idea of this compact transmission system was first introduced [1]. More recently, a vacuum foldedwaveguide system has been proposed as an alternative to the loop antennas currently being used for plasma heating in the middle to upper part of the ion-cyclotron range of frequencies (ICRF, 20 to $200 \mathrm{MHz}$ ) [2]. This folded waveguide system includes the use of a polarizing plate which is designed to increase the on-axis radiation from the launcher and reduce the strength of field components with undesired polarizations; and a sliding-short (or sliding-feedpoint) tuning mechanism to reduce the amount of power refiected from the mismatch between the folded

Manuscript received January 29, 1990; revised May 24, 1990. This work was supported by the U.S. Department of Energy through Grant FG-0286ER53218, and by the University of Wisconsin Graduate School.

The authors are with the Department of Electrical and Computer Engineering, University of Wisconsin, 1415 Johnson Drive, Madison, WI 53706 .

IEEE Log Number 9038631. waveguide coupler and the low plasma surface impedance. In addition, sliding-short tuning is expected to aid in coupling to the fast magnetosonic mode during ICRF heating which requires that the folded waveguide has an electric field minimum near its aperture.

Present coil antennas produce power densities in the 0.3 to $1 \mathrm{~kW} / \mathrm{cm}^{2}$ range, whereas at least $2 \mathrm{~kW} / \mathrm{cm}^{2}$ is required for the success of future fusion devices such as the Compact Ignition Torus (CIT). Results from lower hybrid heating ( $800 \mathrm{MHz}$ to $3 \mathrm{GHz}$ ), in which rectangular waveguides were used to couple an average power of $1 \mathrm{MW}$ with a power density of 5 to $10 \mathrm{~kW} / \mathrm{cm}^{2}$ [4], indicate the viability of waveguide couplers for plasma heating. Coupling in the ICRF on current fusion machines, however, invokes severe size restrictions on the coupling structure; consequently, the examination of compact waveguides such as the dielectric-filled waveguide [3], [5], [6] and the folded waveguide [2], [7] has been carried out.

For example, the plasma surface impedance for a rectangular dielectric-filled $\left(\epsilon_{r}=81\right)$ waveguide has been theoretically calculated to be about $10+j 10 \Omega$ for second-harmonic heating of deuterium at $55 \mathrm{MHz}$ and assuming various density profiles with reactor-like tokamak parameters: Major radius $=480 \mathrm{~cm}$; minor radius $=130$ $\mathrm{cm}$; magnetic field at center $=36 \mathrm{kG} ; 50-50 \%$ deuterium-tritium plasma with density at center $=1.5 \times 10^{14}$ $\mathrm{cm}^{-3}$ and a $1 \%$ edge density [3]. However, the dielectric filling must be able to withstand high neutron fluxes, and its viscosity (or rigidity in the case of a solid) makes sliding-short tuning extremely difficult. The use of vacuum folded waveguide techniques obviates the need for reactor-tolerant dielectrics and readily permits sliding-short or sliding-feedpoint tuning methods

Several aspects of the folded waveguide are examined in this paper. Section II discusses the use of a numerical approach (using Ritz's variational method) to determine folded-waveguide cutoff wavenumbers and electromagnetic field structures. Comparisons of these results with those obtained using an unfolded equivalent rectangular waveguide model, and between measured cavity resonances and those obtained using the unfolded equivalent waveguide, indicate the feasibility of using this model to simplify the theoretical analysis of various folded waveguide properties. Section III briefly examines a theoretical technique used to determine the reflection coefficient due to an inductive strip placed inside a folded waveguide. These results are used to verify the impedance and reflec- 
tion coefficient analyses, since the same techniques are also used to determine the scattering properties of the polarizing plates. Variational techniques are used to obtain upper and lower bounds for $\left|S_{11}\right|$, and the theoretically obtained limits are shown to be in good agreement with the measured results. In Section IV these methods are used to obtain $\left|S_{11}\right|$ due to a polarizing plate placed in a matched folded waveguide. (The matched folded waveguide is used to provide a well-defined condition so that the polarizing plate scattering coefficient can be accurately determined.) It is found that the theoretically obtained expressions require mathematical transformations in order to accelerate their convergence. The numerical results are then shown to be in good agreement with the measured values of $\left|S_{11}\right|$. Section $\mathrm{V}$ examines the freespace far-field radiation patterns of both an open-ended folded waveguide and one which is terminated with a polarizing plate. These far-field radiation patterns allow one to determine the mode content of the fields within the folded waveguide and the approximate pattern of the radiation fields in the intermediate zone of the waveguide aperture. Consequently, these measurements provide a better understanding of the folded-waveguide excitation fields and how they would couple to a tokamak plasma surface. Radiation patterns from various waveguide field polarizations are measured and these results compare well with theoretically obtained patterns using the equivalent unfolded rectangular waveguide model. Also, relative power measurements obtained during the radiation-pattern experiments allow comparisons between maximum radiated powers for several cases and the results are discussed. Finally, Section VI summarizes the many results obtained in Sections II through V and briefly discusses their significance.

\section{Characterizing the Folded Waveguide}

The complicated boundary conditions of the foldedwaveguide structure make it very difficult to develop a simple analytical theory describing the electromagnetic fields within it; hence theoretical examinations of the folded waveguide typically rely on numerical methods of analysis such as variational or finite-element techniques. As a simplification of its analysis, we show that the folded waveguide can, in many applications, be modeled as a rectangular waveguide of appropriate cross section so that simple analytical techniques can be employed to analyze it. One of the primary goals of this work is to show that this is indeed a legitimate procedure; that is, to show that the folded waveguide can in several cases be modeled as a simple unfolded rectangular waveguide of appropriate cross section and to show that the theoretical results from this model agree with experimentally obtained data.

The cutoff wavelength for a waveguide structure depends on the waveguide cross section, particularly on the "long", waveguide dimension as shown in Fig. 1 and designated as $a^{\prime}$ for the folded waveguide and $a$ for the unfolded equivalent waveguide. We begin our analysis by assuming that the folded waveguide can be represented as

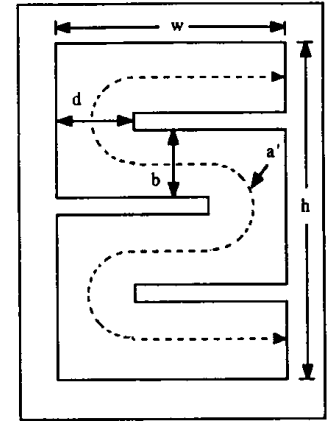

(a)

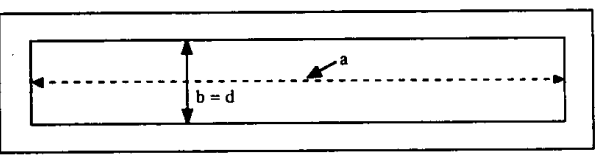

(b)

Fig. 1. (a) The folded waveguide, and (b) the unfolded equivalent. The scale model folded waveguide has $w=1.8 \mathrm{~cm}, h=3.0 \mathrm{~cm}$, a rounded curve path length $a^{\prime}=6.65 \mathrm{~cm}$, and $b=d=0.615 \mathrm{~cm}$. The equivalent unfolded waveguide has a length $a=6.24 \mathrm{~cm}$.

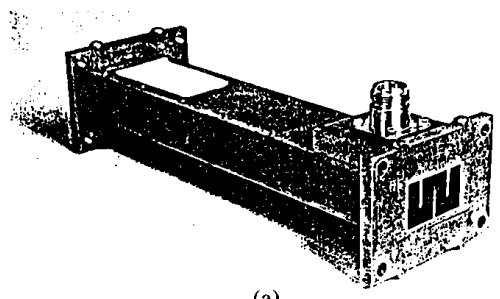

(a)

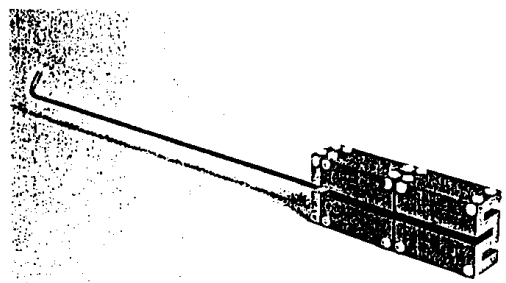

(b)

Fig. 2. (a) Scale model of the ICRF folded waveguide with an overall length of about $20 \mathrm{~cm}$, and (b) the quarter-wave noncontacting slidingshort constructed with the aid of electronic discharge machining (EDM).

an unfolded rectangular waveguide of width $a$. Our folded waveguide shown in Fig. 2 (due to the large size and expense of an ICRF folded waveguide-at $100 \mathrm{MHz}$ a folded waveguide would have an approximate cross section of $0.5 \times 0.8 \mathrm{~m}$ and length of over $2 \mathrm{~m}-$ we have examined a scale model) has a rounded curve path length $a^{\prime}=6.65$ $\mathrm{cm}$ and $b=0.615 \mathrm{~cm}$. From the theory of rectangular waveguides, the cutoff wavenumber is given by $k_{c m n}=$ $2 \pi / \lambda_{c m n}=\left[(m \pi / a)^{2}+(n \pi / b)^{2}\right]^{1 / 2}$, where $\lambda_{c m n}$ is the cutoff wavelength of the $m n^{\prime}$ th mode, $a$ is the waveguide width, $b$ is the waveguide height, and $m, n=0,1,2$, .. , with $m$ and $n$ not simultaneously equal to zero. The 
fundamental mode is that which has the smallest cutoff wavenumber; thus $m=1$ and $n=0$. Setting $a=a^{\prime}$, we find that $k_{c 10}=0.472 \mathrm{~cm}^{-1}$.

To check this result we used folded-waveguide cavity measurements and rectangular waveguide cavity theory to experimentally determine $a$. Rectangular waveguide cavity theory provides the result that cavity resonances will occur at the frequencies $f_{m n p}=(c / 2)\left[(m / a)^{2}+(n / b)^{2}\right.$ $\left.+(p / l)^{2}\right]^{1 / 2}$, where $c$ is the speed of light, $p$ is a nonzero integer, and $l$ is the cavity length. Excitation of $f_{10 p}$ resonances with several values of $p$ and $l$ allows us to determine an average measured value of $a=6.24 \mathrm{~cm} \mathrm{[6].}$ Using this result we find that $k_{c 10}=0.504 \mathrm{~cm}^{-1}$, which corresponds to $f_{c 10}=2.40 \mathrm{GHz}$. Consequently, we find that the measured cutoff wavenumber is larger than the theoretical curved-path approximate result by $6.8 \%$. This result is comparable to that obtained in reference [1], where the authors used transmission measurements to find that the equivalent unfolded-waveguide cutoff wavenumber is larger than a rounded curve approximate result by about $10 \%$.

A more rigorous theoretical analysis of the folded waveguide has been made possible with a numerical computer code written at the University of Wisconsin [8], which can be used to determine both field quantities and cutoff wavenumbers. This code solves the variational Helmholtz equation for a two-dimensional rectangular waveguide with any number of rectangular perturbations (such as the fins in a folded waveguide), using a finite number of basis functions (Ritz's method, also known as the Rayleigh-Ritz variational method) that are eigenfunctions of the unperturbed rectangular waveguide. The accuracy of the code has been verified by comparing computed cutoff wavenumbers of the double-ridged waveguide to values found in the literature. Our code produces values within $0.9 \%$ of published results for several different modes [8].

The variational code was run using our folded waveguide dimensions to determine the cutoff wavenumbers that correspond to several unfolded waveguide modes. In Table I these results are compared to those of the unfolded waveguide model with the experimentally determined equivalent unfolded waveguide width, $a=6.24 \mathrm{~cm}$. We see that the variational results are within $10 \%$ of the experimentally determined values-we expect an upper-limit approximation when using this variational approach. These results indicate that the folded waveguide can, indeed, be modeled as an unfolded equivalent waveguide with respect to cutoff wavenumbers.

As an example of the dominant mode field distributions in a folded waveguide, we have chosen to present the results obtained for a tenfold (nine fin) folded waveguide designed to be operated at $80 \mathrm{MHz}$, as shown in Fig. 3 (our fourfold waveguide is just a simpler case and the qualitative results presented are readily generalized to the $N$-fold case). The data shown in this figure are obtained from the folded waveguide potential along a path $s$ that runs through the center of the waveguide in the $x_{1}-x_{2}$ plane
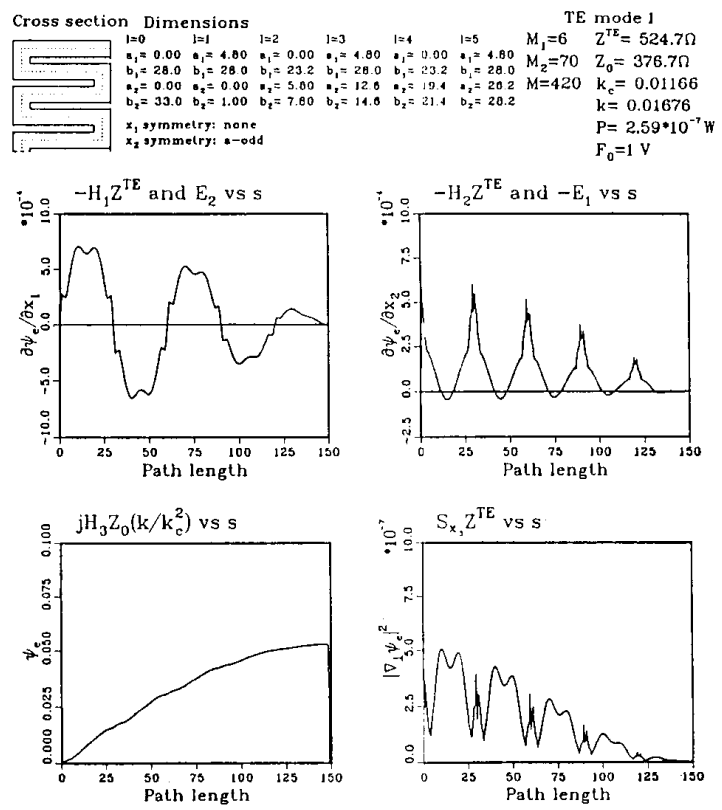

Fig. 3. An example of the field quantities obtained for a tenfold (nine fin) folded waveguide using a numerical analysis based on Ritz's variational technique [8]. A diagram showing half of the tenfold folded waveguide is shown in the upper left, various dimensional parameters in centimeters are shown in the upper middle, and some relevant waveguide quantities are given in the upper right. The four plots show several calculated field quantities, including $E_{1}$ and $E_{2}$ (the horizontal and vertical components of the electric field, respectively), and the power density $S_{x 3}$ along a path $s$ which runs along the center of the folded waveguide (shown as a dotted line in the folded waveguide diagram).

TABLE I

Comparison Between the Cutoff Wavenumbers of THE UNFolded WaVeguide MOdel and the Variational CODE REsults For THE FOLDED GUIDE

\begin{tabular}{llll}
\hline \hline Mode & $\begin{array}{l}\text { Unfolded Model* } \\
\mathrm{k}_{\mathrm{c}}\left(\mathrm{cm}^{-1}\right)\end{array}$ & $\begin{array}{l}\text { Variational Code } \\
\mathrm{k}_{\mathrm{c}}\left(\mathrm{cm}^{-1}\right)\end{array}$ & $\Delta \%$ \\
$\mathrm{k}_{\mathrm{c} 10}$ & 0.504 & 0.496 & -1.6 \\
$\mathrm{k}_{\mathrm{c} 20}$ & 1.01 & $\ldots \ldots . . * *$ &.-- \\
$\mathrm{k}_{\mathrm{c} 30}$ & 1.29 & 1.41 & +9.3 \\
$\mathrm{k}_{\mathrm{c} 40}$ & 2.02 & 2.20 & +8.9 \\
$\mathrm{k}_{\mathrm{c} 50}$ & 2.52 & +6.7 \\
*The value of $k_{c 10}$ listed here was determined by measurements. The values of $k_{c m}$ with \\
$m>1$ have been calculated using an unfolded waveguide model with the dimension $a$ \\
determined as described in the text. \\
**The variational code did not calculate a cutoff wavenumber for this mode. \\
\hline
\end{tabular}

( $x_{1}$ and $x_{2}$ represent the horizontal and vertical axes, respectively). The odd symmetry of the electric potential was utilized so that only half of the waveguide was analyzed as shown.

The alternating polarity of the electric-field component perpendicular to the fins $E_{2}$ (see Fig. 3) indicates that there would be a very small $x_{2}$ component of the radiated farfield for an open-ended folded waveguide. Consequently, the introduction of polarizing plates to limit the $E_{2} \mathrm{com}$ ponent to a single polarity was proposed [2]. On the other hand, the electric-field component parallel to the fins $E_{1}$ is seen to have nearly unidirectional peaks with a mag- 


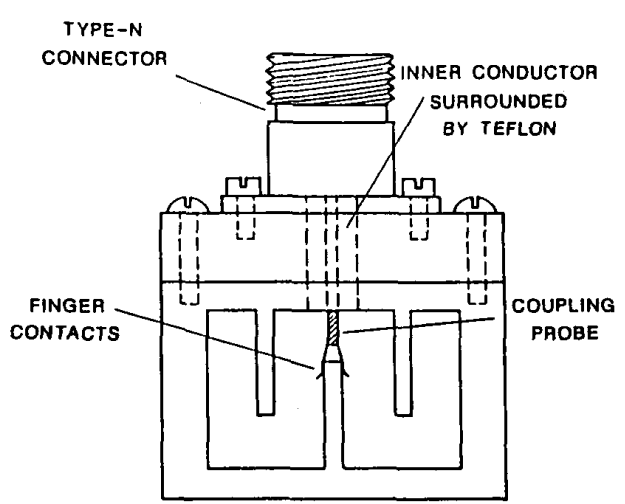

Fig. 4. The coupling configuration of the folded waveguide.

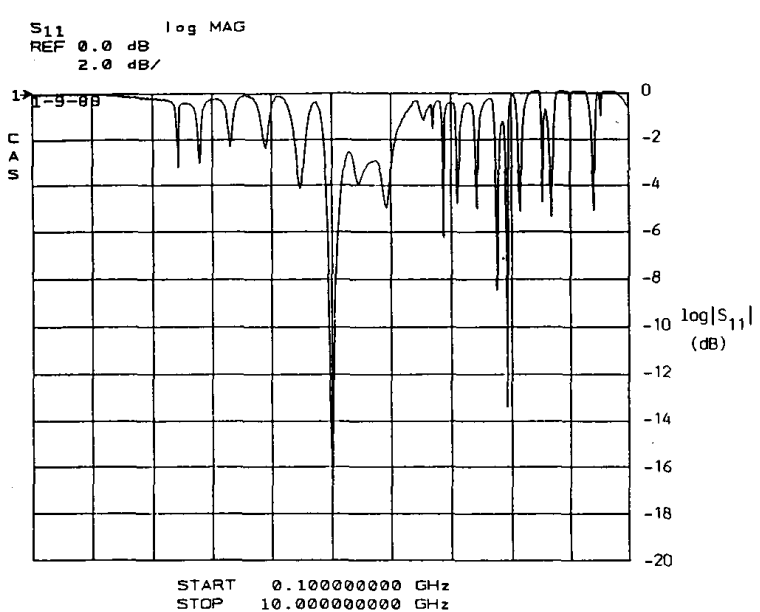

Fig. 5. Cavity measurement of $\left|S_{11}\right|$ scanned over the frequency range 0.1 to $10.0 \mathrm{GHz}$.

nitude comparable to that of $E_{2}$. We show later (in Section IV) that it is $E_{1}$ which provides the largest contribution to the radiated far-field power for the open-ended folded waveguide, and $E_{2}$ provides the largest contribution to the radiated far-field power when a polarizing plate is attached to the aperture.

All of the scattering parameter related experimental results presented in this paper were obtained using an HP8510A network analyzer. The network analyzer was connected to the coupling section of the folded waveguide through an $\mathrm{N}$-connector with finger-contacts attached to the center fin of the waveguide as shown in Fig. 4. Using the coupling section of the folded waveguide with both ends terminated by a shorting plate and the network analyzer calibrated at the $\mathrm{N}$-connector (using a commercial calibration kit), various cavity measurements were performed to determine the resonances of the folded waveguide. The results of the these measurements for our waveguide, with $a=6.24 \mathrm{~cm}, b=0.615 \mathrm{~cm}$, and $l=$ $19.5 \mathrm{~cm}$, are shown in Fig. 5 .

In analyzing these results, a comparison with the unfolded waveguide theory was completed and the results
TABLE II

The Measured Folded-Waveguide Resonances Below $10 \mathrm{GHz}$ and Those Calculated Using an Unfolded Rectangular Waveguide MODEL

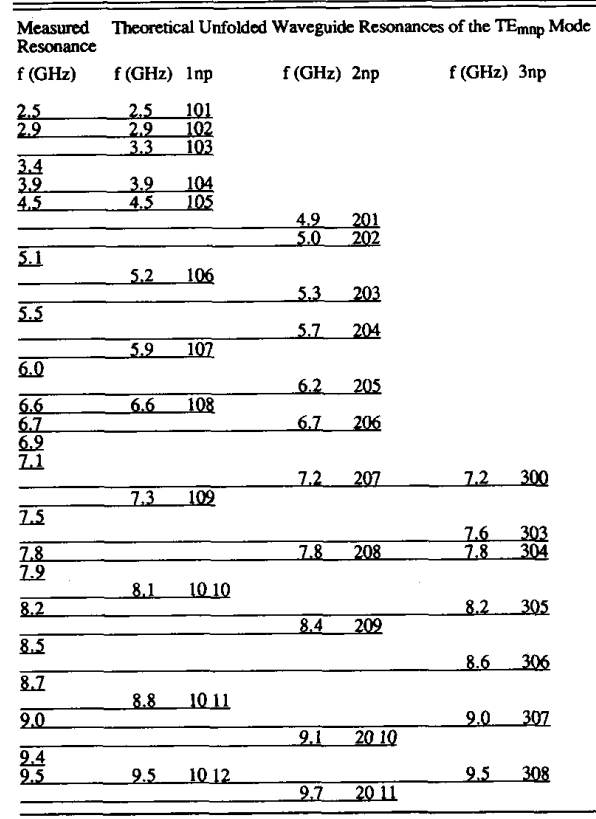

are shown in Table II. The measured resonances listed on the left-hand side of Table II are to be compared with the calculated resonances listed on the right. We find a very good correspondence between the measured resonances and those predicted by the simple unfolded waveguide model, particularly for the resonances at the lower frequencies. The close correspondence between the folded waveguide theory and measurements and those of the unfolded equivalent model confirm our supposition that the folded waveguide can in this case be modeled as an equivalent unfolded rectangular waveguide.

\section{Inductive Strips and Apertures in a Folded WAVEGUIDE}

As mentioned in the introduction, ICRF heating requires coupling to the fast magnetosonic wave in a plasma. In a typical magnetic-confinement fusion device, this entails the launching of fast magnetosonic waves with an electric field $E_{\perp}$ that is perpendicular to the static magnetic field and has a left circularly polarized component at the core that heats the plasma [9]. In the folded waveguide, however, the electric field continuously alters direction as one proceeds around the channels of the waveguide's cross section. This field configuration should result in a radiation pattern with a null on-axis and strong sidelobes, whereas it is desirable to have a radiation pattern which is primarily on-axis. The introduction of a polarizing plate as shown in Fig. 6 produces the desired radiation pattern [2]. The polarizing plate is designed to allow the transmission of $E_{\perp}$ while substantially reducing 


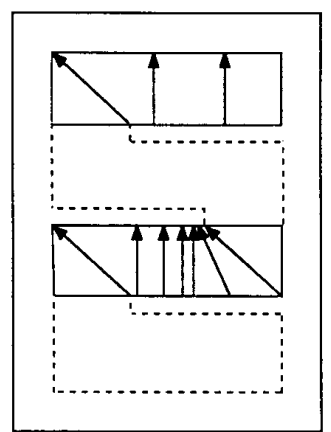

(a)

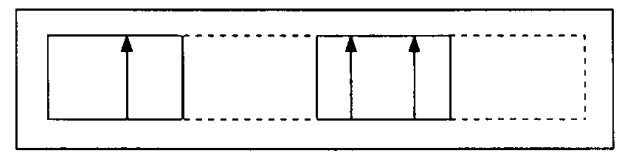

(b)

Fig. 6. Rough, qualitative picture of the electric fields after the attachment of a polarizing plate to (a) the folded waveguide, and (b) the unfolded model.

the orthogonal fields, resulting in modified fields approximately represented by those shown in Fig. 6. The foldedwaveguide launcher can also accommodate a Faraday shield structure to further suppress parallel electric fields if necessary.

The theoretical characterization of the effects of a polarizing plate requires the use of a simplified model for analysis. Our previous success with the unfolded equivalent model indicates its attractiveness for use in modeling the polarizing plates. As shown in Fig. 6, the folded waveguide with a polarizing plate can be modeled as an equivalent unfolded waveguide with conducting strips attached to it; this is the approach we shall take to model the polarizing plates on a folded waveguide. Due to the field structure of the dominant mode in a rectangular waveguide, the attachment of a rectangular strip to the use in the analysis of the polarizing plate. Once the inductive strip reactance is obtained theoretically, we compare the results to those obtained from network analyzer measurements. Since the network analyzer cannot compute phase information for band-limited devices, our quantity of interest is $\left|S_{11}\right|$. From the consideration of a shunt reactance across a transmission line, it is straightforward to calculate the magnitude of the reflection coefficient due to an inductive strip in a rectangular waveguide as

$$
\left|S_{11}\right|=\frac{1}{\sqrt{1+4 x^{2}}}
$$

where the normalized inductance is given by $x=X / Z_{0}$ and $Z_{0}$ is the dominant mode waveguide impedance.

We begin our theoretical analysis with a look at an inductive strip in a rectangular waveguide, shown in Fig. 7(b), and later assume that it is a legitimate model for an inductive strip in a folded waveguide, as shown in Fig. 7(a). We assume that the rectangular guide is infinite in the $\pm z$ directions and that there is an incident $\mathrm{TE}_{10}$ field coming in from minus infinity and impinging on the thin conducting strip, thus allowing scattered and transmitted $\mathrm{TE}_{\mathrm{m} 0}$ fields inside the waveguide. An analysis which includes a summation over waveguide modes at the discontinuity and other standard techniques in electromagnetic theory yields a variational expression for the normalized reactance in terms of integrals over the strip's surface current $[10],[11]$. Since a variational expression produces a second-order error with a first-order error in the trial quantity, we may obtain an estimated value of the normalized inductance close to the actual value (typically an upper bound) using only an approximate expression for the current on the strip. Using the approximate current distribution $\boldsymbol{J}_{s}=\boldsymbol{n} \times \boldsymbol{H}$, where $\boldsymbol{n}$ is a unit vector normal to the strip's surface and $\boldsymbol{H}$ is the unperturbed dominantmode magnetic field of the rectangular waveguide, we find that

$$
x=\frac{\frac{a^{2}}{8 \pi^{2}}\left|\beta_{1}\right| \sum_{m=2}^{\infty} \frac{1}{\beta_{m}}\left(\frac{\sin \left[(m-1) \frac{\pi d}{a}\right]}{(m-1)}-\frac{\sin \left[(m+1) \frac{\pi d}{a}\right]}{(m+1)}\right\}^{2}}{\left[\frac{d}{2}-\frac{a}{4 \pi} \sin \left(\frac{2 \pi d}{a}\right)\right]^{2}}
$$

interior of the guide produces an inductive reactance. The value of this inductance can be calculated using variational techniques [10], [11], and these procedures can be generalized to an arbitrary number of strips in a rectangular waveguide to model the polarizing plates attached to a folded waveguide. The validity of this modeling procedure is subsequently verified by the close correspondence between theoretical and measured results.

We initially examine a single inductive strip to verify both our model and the measurement techniques that we where $\beta_{m}=\left[(m \pi / a)^{2}-k_{o}^{2}\right]^{1 / 2}$. Equation (2) has been evaluated numerically for an inductive strip in a rectangular waveguide and found to converge quite rapidlywithin $10 \%$ of the final value of $x=9.01$ after nine terms for our parameters at $3.0 \mathrm{GHz}$.

An alternative method is what we shall call the inductive aperture technique [10], [11]. In this procedure it is the aperture fields that are of primary consideration, and we will find a variational expression for the admittance of the aperture in a rectangular waveguide which contains an infinitesimally thin conducting strip as shown in Fig. 7(b). 


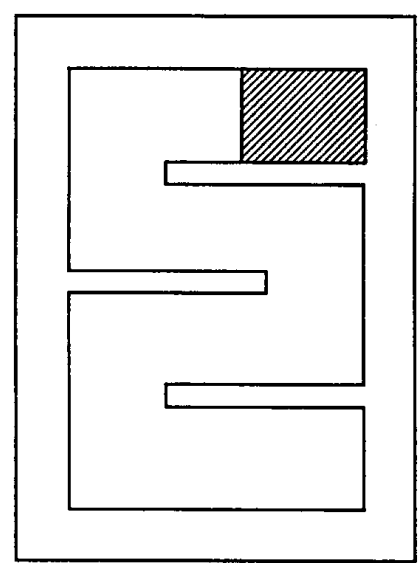

(a)
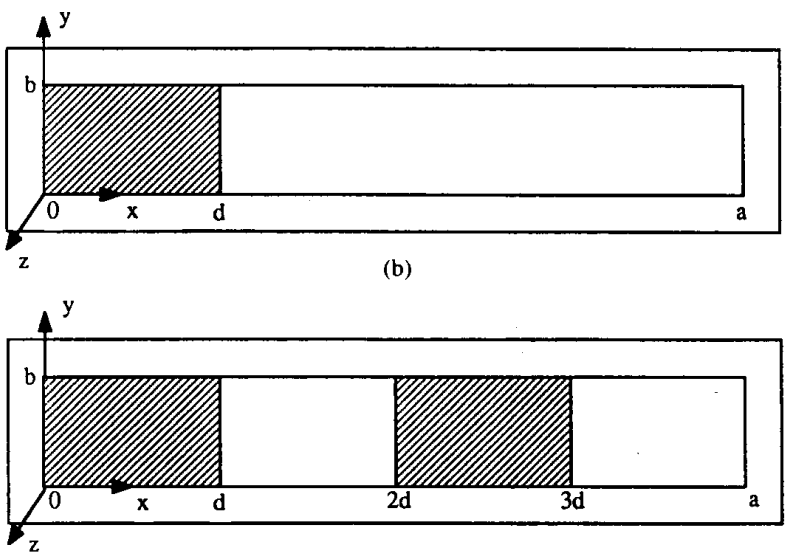

(c)

Fig. 7. Cross sections of (a) a conducting strip in a folded waveguide, (b) a conducting strip of width $d$ (extending from $x=0$ to $x=d$ ) and height $b$ inside a rectangular waveguide of width $a$, and (c) a cross section of the rectangular waveguide with inductive strips used to model the folded waveguide when a polarizing plate is used.

In this case, a source at minus infinity launches $\mathrm{TE}_{10}$ mode fields toward an aperture which lies between $x=d$ and $x$ $=a$, and the consequent interaction leads to both scattered and transmitted $\mathrm{TE}_{\mathrm{m} 0}$ fields. Again, a variational expression is obtained; however, this time it is for the inductive susceptance (as a result, we obtain a lower bound to the value of the inductive reactance), and it is in terms of integrals over expressions which include derivatives of the aperture fields.

We use the unperturbed $T E_{10}$ rectangular waveguide electric field in the aperture (this field does not satisfy the aperture boundary conditions but it does allow easy evaluation) and choose it to be of unity amplitude, $E_{a p}=\sin$ $(\pi x / a)$. We find that:

$$
x=\frac{\frac{2 \pi^{2}}{a^{2}}\left|\beta_{1}\right|\left[\frac{a-d}{2}-\frac{a}{4 \pi} \sin \left(\frac{2 \pi d}{a}\right)\right]^{2}}{\sum_{m=2}^{\infty} \frac{\beta_{m}}{m^{2}}\left\{\frac{\sin [(m-1) \pi]-\sin \left[(m-1) \frac{\pi d}{a}\right]}{m-1}+\frac{\left.\sin [(m-1) \pi]-\sin \left[(m+1) \frac{\pi d}{a}\right]\right\}^{2}}{m+1} .\right.}
$$


the strips is slightly larger than that given by this approximation because the polarizing plates are designed to entirely cover the internal fins). For an odd number of fins in the folded waveguide we have an even number of inductive strips $N$ when using the unfolded rectangular waveguide model. Since the calculation of the inductance requires an integration over each of the strips, we end up with a summation of terms which has the form:

$$
\begin{aligned}
\left.f(x)\right|_{S}= & \left.f(x)\right|_{x=0} ^{a / N}+\left.f(x)\right|_{2 a / N} ^{3 a / N}+\left.f(x)\right|_{4 a / N} ^{5 a / N} \\
& +\cdots+\left.f(x)\right|_{(N-1) a / N} ^{a} \\
= & \left.\sum_{s=1}^{N / 2} f(x)\right|_{(2 s-1) a / N} ^{a}
\end{aligned}
$$

when generalizing (2) to the $N$ symmetric strips case. This leads to

$$
\begin{aligned}
& x= \\
& \sum_{m=2}^{\infty} \frac{1}{\beta_{m}}\left\{\sum _ { s = 1 } ^ { N / 2 } \left\{\operatorname{sinc}\left(\frac{m-1}{2 N}\right) \cos \left[\frac{\pi(m-1)(4 s-3)}{2 N}\right]\right.\right. \\
&\left.\left.-\operatorname{sinc}\left(\frac{m+1}{2 N}\right) \cos \left[\frac{\pi(m+1)(4 s+3)}{2 N}\right]\right\}\right\}^{2}
\end{aligned}
$$$$
\frac{2}{\left|\beta_{1}\right|}\left\{\sum_{s=1}^{N / 2}\left\{1-\operatorname{sinc}\left(\frac{1}{N}\right) \cos \left[\frac{\pi(4 s-3)}{N}\right]\right\}\right\}^{2}
$$

where $\operatorname{sinc}(x)=(1 / \pi x) \sin (\pi x)$ and there are $N / 2$ conducting strips with $N$ taken to be even for our caseit is straightforward to examine the case in which there is an odd number of strips or apertures using the same techniques. It can be shown that (5) is equivalent to that found by Lee [6]. This equation has been evaluated numerically and has been found to be divergent for our parameters.

The inductive aperture technique, which evaluates the impedance of a polarizing plate in a folded waveguide by modeling it as an unfolded waveguide with a series of inductive apertures inside it, is considered next. Since we obtained good results for the inductive aperture technique applied to the single inductive strip using the unperturbed $\mathrm{TE}_{10}$ waveguide field, we also use this field for our polarizing plate evaluation. An examination of the resulting equations provides an expression which is quite similar to the inverse of (5) with the signs of certain terms changed. The result is:

$$
\begin{aligned}
& x= \\
& \frac{\left|\beta_{1}\right|}{2}\left\{\sum_{s=1}^{N / 2}\left\{1+\operatorname{sinc}\left(\frac{1}{N}\right) \cos \left[\frac{\pi(4 s-3)}{N}\right]\right\}\right\}^{2} \\
& \sum_{m=2}^{\infty} \frac{\beta_{m}}{m^{2}}\left\{\sum _ { s = 1 } ^ { N / 2 } \left\{\operatorname{sinc}\left(\frac{m-1}{2 N}\right) \cos \left[\frac{\pi(m-1)(4 s-3)}{2 N}\right]\right.\right. \\
& \left.\left.+\operatorname{sinc}\left(\frac{m+1}{2 N}\right) \cos \left[\frac{\pi(m+1)(4 s+3)}{2 N}\right]\right\}\right\}^{2}
\end{aligned}
$$

where $N$ is again taken to be even for our case. Equation (6) has also been numerically evaluated and the results of a convergence test indicate very slow convergence-even after the evaluation of $3 \times 10^{5}$ terms.

As a consequence of this slow convergence, we employ a mathematical transformation which will allow us to develop a more rapidly converging expression. Following Schwinger's transformation procedure [12], we have generalized the single inductive aperture equation to the case of $N / 2$ waveguide apertures (with $N$ even for our evaluation). We find that

$$
\begin{aligned}
& x \approx \frac{a}{2 \pi}\left|\beta_{1}\right|\left[\sum_{s=1}^{N / 2} \sin \left(\frac{\pi}{2 N}\right) \sin \left[\frac{\pi}{2 N}(4 s-1)\right]\right]^{2} \\
& \sum_{s=1}^{N / 2}\left\{1-\sin ^{2}\left(\frac{\pi}{2 N}\right) \sin ^{2}\left[\frac{\pi}{2 N}(4 s-1)\right]\right. \\
& \left.+\sum_{m=2}^{\infty}\left[\left(\frac{\frac{a}{\pi} \beta_{m}-m}{m^{2}}\right) P_{m 1}^{2}\right]\right\}
\end{aligned}
$$

where we have used the relationship $d=a / N$ in the sine terms (this is specific to our geometry since $N$ is equal to the number of strips plus the number of apertures) and the $P_{m 1}$ 's are constant coefficients that are yet to be determined. Equation (7) is our final, rapidly converging expression for the multiple aperture inductance.

The coefficients $P_{m 1}$ are determined from

$$
\cos \left(\frac{m \pi x}{a}\right)=T_{m}\left[\cos \left(\frac{\pi x}{a}\right)\right]=\sum_{n=0}^{m} P_{m n} \cos (n \theta)
$$


where the $T_{m}[\cos (\pi x / a)$ ]'s are Chebyshev polynomials and $\theta$ is our transformation variable. The generalization of Schwinger's transformations to the case of $N / 2$ waveguide apertures (with $N$ even for our evaluation) requires that we take:

$$
\begin{aligned}
\cos \left(\frac{\pi x}{a}\right)= & \cos \left[\frac{\pi d}{2 a}\right] \cos \left[\frac{\pi d}{2 a}(4 s-1)\right] \\
& +\sin \left[\frac{\pi d}{2 a}\right] \sin \left[\frac{\pi d}{2 a}(4 s-1)\right] \cos \theta
\end{aligned}
$$

where $s$ is an integer and $1 \leq s \leq N / 2$. When the Chebyshev polynomials are written explicitly in terms of cos ( $n \theta)$ using (9), $P_{m 1}$ is the coefficient of the $\cos (\theta)$ term. The coefficients $P_{m 1}$ have been calculated up to $m=12$ and used in a numerical evaluation of (7). The results of a convergence test for our parameters at $3.0 \mathrm{GHz}$ yields values within $10 \%$ of the final value of $x=5.86 \times 10^{-2}$ after only three terms are summed (we used 11 terms for our evaluations to get within $0.022 \%$ of the value calculated with the use of the 12 th term). This provides us with a lower limit approximation to the actual value of the normalized inductance and, consequently, an upper limit to $\left|S_{11}\right|$ as found using (1).

Reflection coefficient measurements have been made using polarizing plates inserted between the coupling and matched sections of the folded waveguide. Calibration of the network analyzer for these measurements required that we produce our own standards including a shorting plate, a folded waveguide offset short, and a matched load that was constructed by inserting a tapered piece of Eccosorb LS-30 along each of the fins inside a folded waveguide section (a measured return loss of greater than $20 \mathrm{~dB}$ over the desired frequency range verifies the effectiveness of this construction). The time domain result from the network analyzer measurements, which also shows the gating window, is presented in Fig. 9. (The gating window is used to isolate the desired time-domain response by filtering out the unwanted signal which lies outside the window.) A comparison between the measurements (both gated and ungated responses) and the aperture theory are shown in Fig. 10. Again, very good results are obtained even though the theory does not predict the apparent oscillatory nature of the measured results. The theoretical results do, however, provide a reasonably close upper limit - the worst correspondence between the theory and gated measurement occurs at $4.5 \mathrm{GHz}$, where they differ by $2.9 \%$. Hence the validity of modeling the polarizing plates in a matched folded waveguide as symmetric inductive apertures in an equivalent rectangular waveguide has been verified.

The theoretical evaluation of the polarizing plate reflection coefficient assumed the plate to be of negligible thickness. Consequently, a measurement comparing polarizing plates of different thickness was performed. Once again, each polarizing plate was attached between the

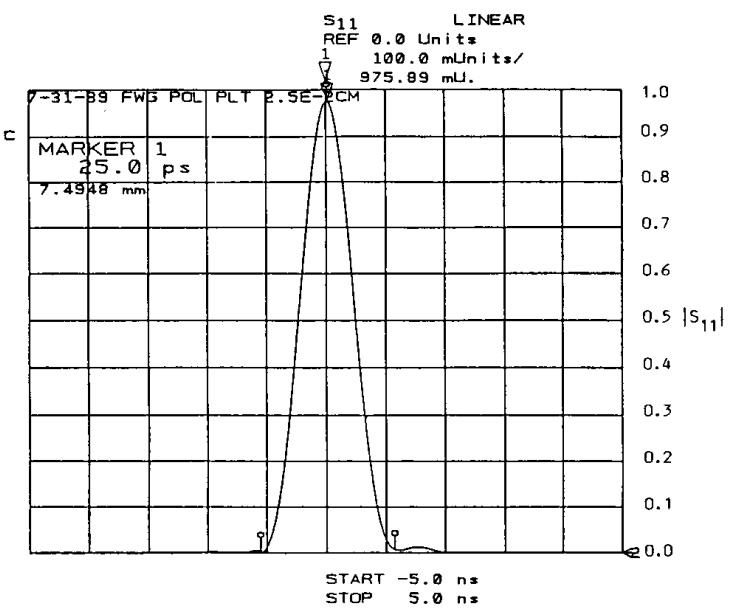

Fig. 9. The time domain response of a polarizing plate in a folded waveguide showing the gated region which lies between the small markers ( $-6 \mathrm{~dB}$ points $)$. The thickness of the polarizing plate is $2.5 \times 10^{-2} \mathrm{~cm}$.

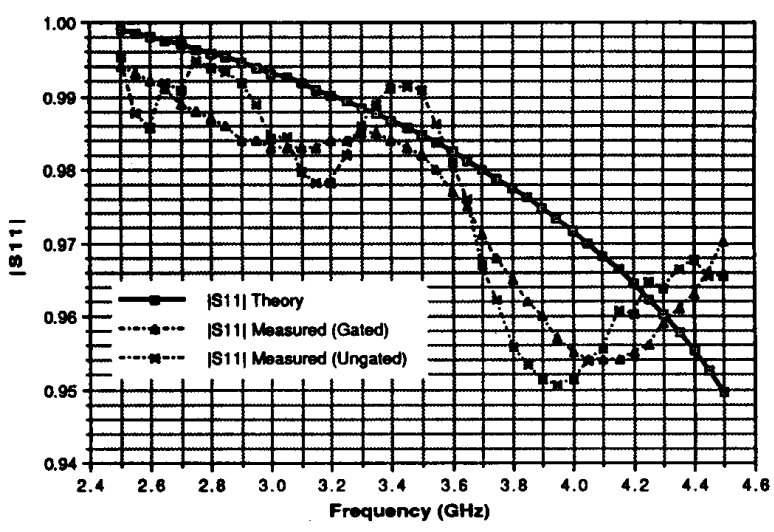

Fig. 10. Comparison between theoretical and experimental polarizing plate data. The aperture theory used 11 terms and provides an upper bound for $\left|S_{11}\right|$ (lower bound for $X / Z_{0}$ ). The theoretical evaluation neglects the polarizing plate thickness of $0.025 \mathrm{~cm}$.

coupling and matched sections of the folded waveguide for the measurements. The comparison of two plates that differ in thickness by a factor of 12.8 is shown in Fig. 11 . This plot indicates little difference between the two measurements except at high frequencies where the discrepancy may be due to imperfect electrical contact (the rigidity of the thicker plate makes it less likely to conform with surface variations along the contacting regions between the folded waveguide sections).

Measurements have also been made using a slidingshort, which is used to maximize the power coupled through the polarizing plate to a folded waveguide matched load attached to the opposite end. Network analyzer measurements over the frequency range 2.5 to 3.5 $\mathrm{GHz}$ with the calibration plane set at the folded wave- 


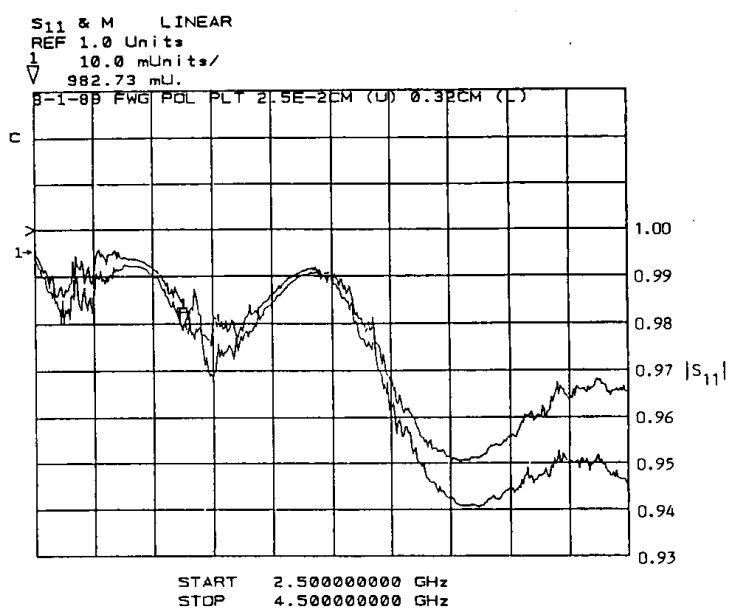

Fig. 11. Comparison of the reflection coefficients $\left|S_{11}\right|$ of a polarizing plate which is $0.025-\mathrm{cm}$ thick (upper) to one that is $0.32-\mathrm{cm}$ thick (lower) as a function of frequency.

guide-polarizing plate interface indicate that a substantial reduction of $\left|S_{11}\right|$ occurs when the sliding-short is positioned such that a cavity resonance occurs. Since tuning to a waveguide cavity resonance increases the cavity field magnitudes, more power is radiated from the polarizing plate slots than in the detuned case, leading to a lower reflection coefficient.

Using sliding-short tuning during reflection coefficient measurements, we have found that greater than $99 \%$ power can be transmitted through the folded waveguide terminated with a matched load, and over $95 \%$ power can be transmitted through the folded waveguide terminated with a polarizing plate backed by a matched load (the fraction of power transmitted $=1-\left|S_{11}\right|^{2}$ ). From these measurements, we infer that the radiation efficiency can be made quite high for radiation into a plasma. We conclude that sliding-short tuning is effective as a means of maximizing the coupled power when using polarizing plates attached to a folded waveguide.

\section{Radiated Power and Patterns}

Measurements of the far-field radiation patterns of the open-ended folded waveguide and the folded waveguide with a polarizing plate attached have been obtained in several planes of field polarization. We have taken the primary polarization of the folded waveguide radiation field to be defined by the polarization of the largest magnitude electric field component of the open-ended folded waveguide-which lies in the plane of the central fin for our folded waveguide. This convention is taken so that when our waveguide is "unfolded," the field polarization agrees with the terminology commonly used to describe the radiation from rectangular waveguides; that is, the $\mathrm{H}$ and $E$-plane radiation fields. Consequently, our measure-

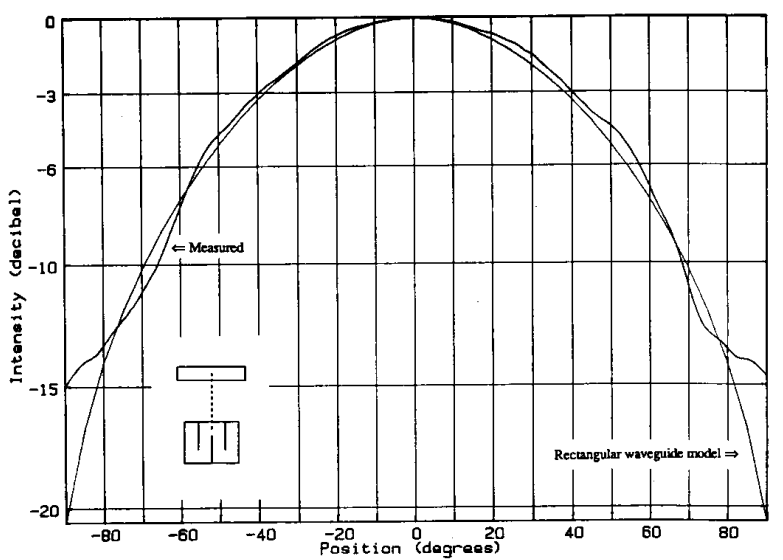

(a)

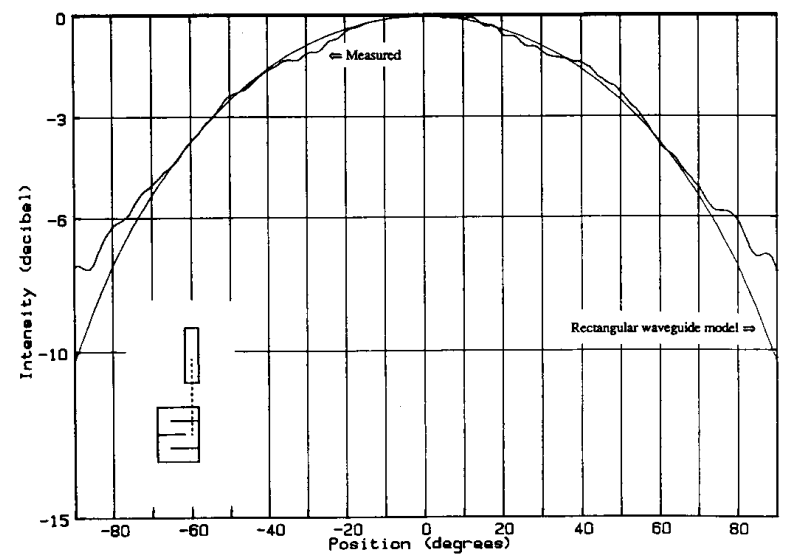

(b)

Fig. 12. (a) Co-polarized, $H$-plane, open-ended folded waveguide far-field radiation measurements with a comparison to the equivalent unfolded waveguide radiation pattern with $\Gamma_{\phi}=0.58$, and (b) co-polarized, $E$-plane, open-ended folded waveguide far-field radiation measurements with a comparison to the equivalent unfolded waveguide radiation pattern with $\Gamma_{\theta}=-0.58$

ment system was aligned so that the point of rotation of the receiving horn was located midway between the central folded-waveguide fin and the waveguide wall across from it. (See the schematic diagrams on Figs. 12 and 13 for an indication of how co- and cross-polarization apply to our folded waveguide-receiving horn system.)

The radiation measurements were performed using a 3.0-GHz backward-wave oscillator capable of delivering $75 \mathrm{~mW}$ to our folded waveguide. The folded waveguide was attached to a table and radiated into an anechoic chamber containing an exponentially tapered horn (aperture dimensions of $23.15 \times 17.15 \mathrm{~cm}$ ) which was placed on a moveable beam about $130 \mathrm{~cm}$ away $\left(2 D^{2} / \lambda \approx 8 \mathrm{~cm}\right.$ for the folded waveguide, hence the far-field condition is easily satisfied ) and was rotated about the alignment center of the folded waveguide. A wall of microwave absorbent material was placed behind the folded waveguide 


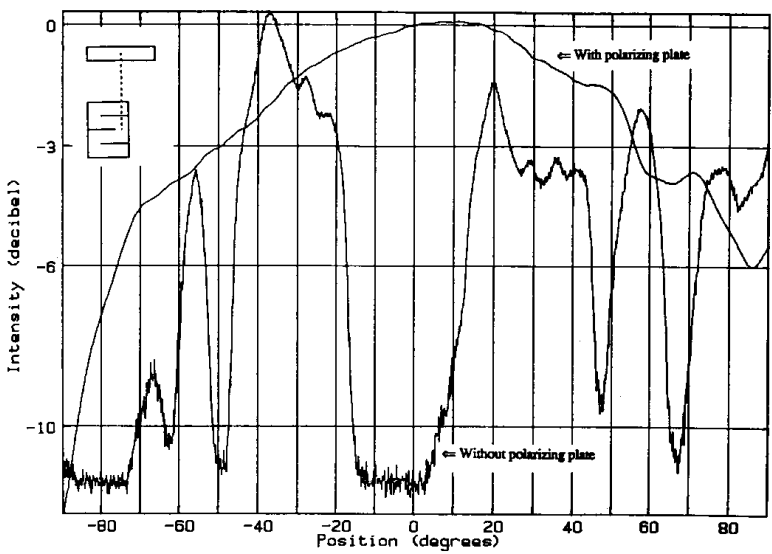

(a)

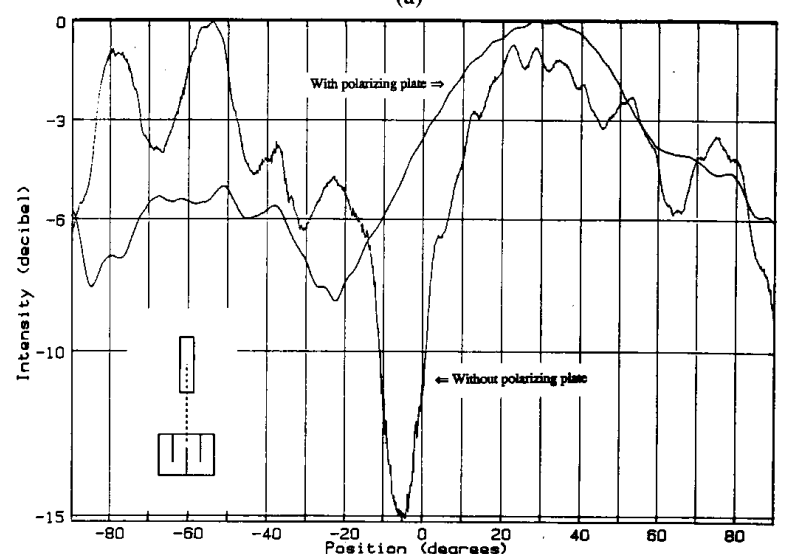

(b)

Fig. 13. (a) Cross-polarized, $H$-plane, and (b) cross-polarized, $E$-plane farfield radiation patterns of the folded waveguide both with and without a polarizing plate of thickness $0.025 \mathrm{~cm}$ attached to the aperture.

aperture flange (as seen in Fig. 2) to reduce the reception of undesired signals, and we attached our quarter-wave sliding-short behind the coupling section to allow tuning for maximum radiated power. The power received by the horn was fed into a crystal detector and connected to a swept amplitude analyzer. The resulting signal was then converted to a voltage between $\pm 5 \mathrm{~V}$, depending on the intensity of the signal, and stored in a computer which monitored and/or controlled the various data collection and analysis systems. The measured data was corrected and linearized using calibration data obtained before each run. Note that, due to chamber wall reflections, data taken beyond $\pm 80^{\circ}$ must be considered questionable.

Results from our measurements are presented in Figs. 12 and 13. A comparison between the co-polarized folded waveguide $H$ - and $E$-plane radiated fields and those of the theoretical dominant-mode patterns of the equivalent unfolded rectangular waveguide is shown in Fig. 12(a) and (b), respectively. Here we have utilized the results of [13] for the rectangular waveguide electric fields in the far- field for the $E$-plane:

$$
\begin{aligned}
E_{\theta}= & \frac{2 a^{2} b}{\pi \lambda^{2} R}\left(\frac{\mu}{\epsilon}\right)^{1 / 2}\left[1+\frac{\beta_{10}}{k} \cos \theta\right. \\
& \left.+\Gamma_{\theta}\left(1-\frac{\beta_{10}}{k} \cos \theta\right)\right] \\
& \times \frac{\sin \left(\frac{\pi b}{\lambda} \sin \theta\right)}{\frac{\pi b}{\lambda} \sin \theta} e^{-j k R}
\end{aligned}
$$

and for the $H$-plane:

$$
\begin{aligned}
E_{\phi}= & -\frac{a^{2} b}{2 \pi \lambda^{2} R}\left(\frac{\mu}{\epsilon}\right)^{1 / 2}\left[\cos \theta+\frac{\beta_{10}}{k}\right. \\
& \left.+\Gamma_{\phi}\left(\cos \theta-\frac{\beta_{10}}{k}\right)\right] \\
& \times \frac{\cos \left(\frac{\pi a}{\lambda} \sin \theta\right)}{\left(\frac{\pi a}{\lambda} \sin \theta\right)^{2}-\frac{\pi^{2}}{4}} e^{-j k R}
\end{aligned}
$$

where $R$ is the distance from the waveguide alignment center, $\theta$ is the angle measured from the alignment axis, and the $\Gamma_{\theta, \phi}$ are the aperture reflection coefficients, which we take as empirical parameters to be determined from the radiation pattern measurements. It should be noted that (10) and (11) are obtained using approximations that limit the accuracy of the results for $a / \lambda, b / \lambda<1$, whereas the equivalent rectangular model for our folded waveguide has $a / \lambda=0.624$ and $b / \lambda=0.0616$. We find, however, that these co-polarization measurements of the folded-waveguide radiation fields are described very well by the rectangular waveguide model when the aperture reflection coefficients are determined by comparison between the measurements and theory to be $\Gamma_{\theta}=-0.58$ and $\Gamma_{\phi}=0.58$. In addition, the maximum co-polarized field powers for the open-ended waveguide were measured to be more than $25 \mathrm{~dB}$ above the open-ended cross-polarized field powers. Finally, we note that tuning with the slidingshort was useful in adjusting the radiated power for these and the following measurements, and all presented results were obtained with the tuning adjusted for maximum detected power.

Next, the effects of attaching a polarizing plate to the folded-waveguide aperture were observed. The resulting $H$ - and $E$-plane measurements, which correspond to the cross-polarized fields according to our definition, are shown in Fig. 13(a) and (b). Results closely match those anticipated by Owens [2] and Lee [6] in their theoretical analyses of the $E$-plane fields. These results reveal the increased directivity of the cross-polarized field patterns with the use of a polarizing plate in comparison to the 


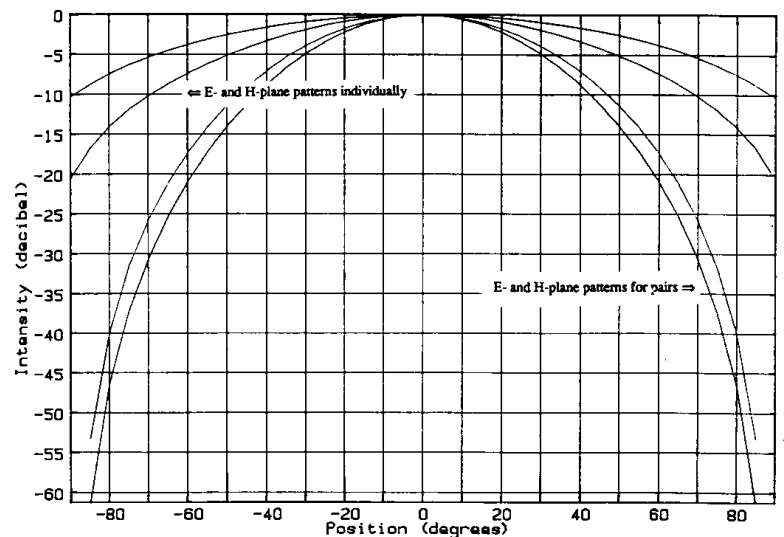

Fig. 14. A comparison of the theoretical co-polarized $E$ - and $H$-plane radiation patterns of a single open-ended folded waveguide, and a phasematched pair of open-ended folded waveguides placed $\lambda / 2$ apart using the equivalent unfolded rectangular waveguide model.

open-ended cases. The maximum detected cross-polarized powers with the polarizing plate attached is about $5 \mathrm{~dB}$ above the measured co-polarized powers using the polarizing plate, and about $10 \mathrm{~dB}$ above those obtained without the polarizing plates. Note, however, that the maximum detected power for the cross-polarized case with the polarizing plate attached is about $17 \mathrm{~dB}$ below the open-ended co-polarized field powers. This is due to the large aperture reflection coefficient produced by the polarizing plate when coupled to free space. Coupling to the low impedance load presented by a plasma surface in the near-field region of the waveguide aperture should permit more radiated power with proper tuning of the slidingshort.

We have found that the open-ended co-polarized radiation patterns (both $H$ and $E$-plane) and the $E$-plane crosspolarized radiation pattern of the polarizing plate case are very similar to those of the unfolded equivalent rectangular waveguide. Simple rectangular waveguide analysis allows us to calculate the dominant mode field radiation patterns of the folded waveguide in these cases; thus, for example, we may easily determine the increase in directivity obtained when using a two-folded waveguide phasematched array spaced a half-wavelength apart. Theoretical radiation patterns can be calculated using the aforementioned rectangular waveguide theory with the electric fields multiplied by the array factor $2 \cos (\pi / 2 \sin \theta)$. The results from this analysis, along with the results from the equivalent unfolded rectangular waveguide as shown previously in Fig. 12(a) and (b), are presented in Fig. 14 for comparison.

\section{Summary and Conclusions}

In the cases considered, we have demonstrated that an equivalent unfolded rectangular waveguide can be used to model the folded waveguide operated in its dominant mode. Our measurements of the width of this equivalent rectangular waveguide indicate that $k_{c 10}$ for the folded waveguide is about $7 \%$ larger than that calculated using a simple curve measured midway between the folds of the waveguide. We have also compared our experimental results to those obtained from a computer code based on Ritz's variational method for the analysis of waveguides of arbitrary cross section and found that the theoretical $k_{c 10}$ differed from the measured value by less than $2 \%$ for the dominant mode and by less than $10 \%$ for several of the higher order modes. The dominant mode folded waveguide, electric-field distributions obtained from our code provided motivation for the use of polarizing plates as a method of increasing the directivity of the radiated power and was useful for obtaining a qualitative understanding of the radiated field patterns.

Next, we examined the effects of an inductive strip in a folded waveguide as a means of establishing theoretical and experimental techniques to be used in the analysis of the polarizing plates. Variational techniques providing expressions for the upper and lower limits to $\left|S_{11}\right|$ for an inductive strip in a matched equivalent rectangular waveguide were found to be in good agreement with measured values over a range of frequencies. As a result, the degree of tuning to a plasma impedance with the sliding-short can be determined by modeling the plasma impedance inside a folded waveguide section using an appropriately sized and located inductive strip (this has been done for the rectangular waveguide [3], [6]).

Application of the variational techniques to a polarizing plate in a matched folded waveguide led to a rapidly converging expression for the upper limit of $\left|S_{11}\right|$. This theoretical upper limit to $\left|S_{11}\right|$ due to the polarizing plate was within $3 \%$ of the measured values over a $2 \mathrm{GHz}$ bandwidth. Consequently, we have verified a theoretical evaluation of the polarizing plate scattering properties-a result which may be useful in modeling folded waveguide launcher-tokamak plasma systems for optimizing the power coupling in such configurations.

An examination of the open-ended, folded waveguide, radiation patterns indicated that the co-polarized fields (those parallel to the folded waveguide fins) are much stronger than had been anticipated. Although polarizing plates are effective at increasing the cross-polarized field directivities, the maximum detected power was found to be about $17 \mathrm{~dB}$ below the maximum open-ended co-polarized power. In addition, the polarizing plate radiation pattern was found to be very similar to that of the openended folded waveguide $E$-plane measurement (which is less directive than the open-ended $H$-plane pattern). The open-ended co-polarized field patterns provided detected powers more than $25 \mathrm{~dB}$ above the orthogonal polarization components, whereas the use of a polarizing plate led to cross-polarized field patterns with detected powers of about $5 \mathrm{~dB}$ above the orthogonal polarization components.

It was also found that we can model the open-ended, co-polarized radiation patterns of the folded waveguide using an equivalent unfolded rectangular waveguide operated in its dominant mode, thus leading to simplified 
analyses of folded waveguide radiation systems. The examination of a two-folded waveguide phase-matched array using the unfolded waveguide model provided a theoretical example of the simplifications possible.

The vacuum far-field radiation patterns provide both a quantitative measurement of the mode content of the fields within the folded-waveguide launcher and the approximate spatial intensity of the radiation fields in the intermediate zone of the folded-waveguide launcher. Hence these measurements provide useful information about the folded waveguide. One could use the unfolded waveguide model with the incident modes determined from the farfield measurements to solve an equivalent simplified waveguide-plasma interface problem in tokamak heating. In addition, if the intermediate or far-fields play a significant role in plasma heating, the large transmitted power and spatial form of the far-field pattern of the open-ended folded waveguide indicate that it may be a useful alternative (with the fins oriented perpendicularly to the toroidal magnetic field) to the folded waveguide with a polarizing plate attached to its aperture for coupling to tokamak plasmas.

In conclusion, we have demonstrated the usefulness of modeling the folded waveguide as an equivalent unfolded rectangular waveguide for the determination of several waveguide properties. It is anticipated that these results will improve the understanding of the folded waveguide structure as a means for ICRF heating of plasmas in fusion devices.

\section{ACKNOWLEDGMENT}

The authors would like to acknowledge the work of Dr. J. L. Lee, who designed our test model and carried out initial research for this folded-waveguide project. They also thank Drs. O. Eldridge and N. Lam for their contributions on various waveguide and plasma related topics, and Dr. T. Owens for a critical reading of the manuscript. Finally, they thank Prof. R. J. Vernon and his graduate students M. Kasraian, F. Schleifer, and P. Sealy for the use and support of their microwave radiation measurement system.

\section{REFERENCES}

[1] W. L. Barrow and H. Schaevitz, AIEE Trans., vol. 60, pp. 119-122, Mar. 1941.

[2] T. L. Owens, IEEE Trans. Plasma Sci., vol. 14, pp. 934-936, Dec. 1986.
[3] N. Lam, J. L. Lee, J. Scharer, and R. Vernon, "Analysis of dielectric-filled waveguide coupling to plasmas in the ICRF," IEEE Trans. Plasma Sci., vol. PS-14, pp. 271-276, June 1986.

[4] S. Knowlton et al., "Energy confinement of lower-hybrid currentdriven tokamak plasmas," Phys. Rev. Lett., vol. 57, pp. 587-590, Aug. 1986.

[5] J. L. Lee, J. E. Scharer, and B. M. Jost, "Coupling and loading measurements for a dielectric-filled ICRF waveguide coupler,' IEEE Trans. Plasma Sci., vol. 16, pp. 645-651, Dec. 1988.

[6] J. L. Lee, "Wave coupling and matching of waveguide couplers in the ion cyclotron range of frequencies," Ph.D. thesis, Univ. Wisconsin, Madison, 1988.

[7] G. L. Chen, T. L. Owens, and J. H. Whealton, "Theoretical study of the folded waveguide," IEEE Trans. Plasma Sci., vol. 16, pp. 305-311, Apr. 1988.

[8] E. J. Sigal, "Variational analysis of multiridged waveguides with applications to ICRF heating," Master's thesis, Univ. Wisconsin, Madison, 1987.

[9] J. Scharer et al., "Fokker-Planck calculations for JET ICRF heating scenarios," Nucl. Fusion, vol. 25, no. 4, pp. 435-444, 1985.

[10] R. E. Collin, Field Theory of Guided Waves. New York: McGrawHill, 1960, pp. 315-363.

[11] L. Lewin, Theory of Waveguides. New York: Wiley, 1975, pp. 167179.

[12] J. Schwinger and D. S. Saxon, Discontinuities in Waveguides. New York: Gordon and Breach, 1968, p. 72.

[13] J. R. Risser, in Microwave Antenna Theory and Design, S. Silver, Ed. London: Peregrinus, 1984, pp. 341-347.

Bradley M. Jost was born in Milwaukee, WI, in 1964 . He received the B.S. degree in electrical engineering technology from the Milwaukee School of Engineering in 1986 and the M.S. degree in electrical engineering from the University of Wisconsin-Madison in 1989. He is currently studying nonlinear optical effects in photorefractive crystals for work toward the Ph.D. degree in electrical engineering.

Mr. Jost is a member of Tau Alpha Pi and the American Physical Society.

John E. Scharer was born in Monroe, WI, in 1939. He received the B.S., M.S., and Ph.D. degrees in electrical engineering from the University of California, Berkeley, in plasma physics.

He is a Professor with the Department of Electrical and Computer Engineering, University of Wisconsin, Madison. He has spent research sabbaticals at the CEA Fontenay-aux-Roses, France, in 1970 and at the JET tokamak at Culham, England, in 1983. He has been active with graduate students and scientists in theoretical, computational, and experimental research in the area of linear and nonlinear plasma waves, and heating of fusion plasmas, space plasmas, and lasers. He has also taught and recently started research in the area of free-electron lasers and coherent sources of radiation.

Dr. Scharer is a member of Tau Beta Pi, the American Physical Society, and the Center for Plasma Theory and Computation at the University of Wisconsin. 